\title{
Igualdad económica en el Ecuador
}

\section{Una visión técnica}

La desigualdad económica hace referencia a la forma como se distribuye el ingreso per cápita del hogar dentro de la sociedad, éste es una medida de bienestar económico que capta todos los ingresos corrientes disponibles del hogar y los asigna proporcionalmente para el número de miembros de la familia (INEC, Reporte de Pobreza por Ingresos, junio 2015, p.6). Para medir la desigualdad económica de un país, el indicador más utilizado es el coeficiente de Gini.

\section{Coeficiente de Gini}

Según el Banco Mundial (BM), el coeficiente de Gini es un indicador que mide hasta qué punto la distribución del ingreso (o, en algunos casos, el gasto de consumo) entre individuos u hogares dentro de una economía se aleja de una distribución (por persona) perfectamente equitativa. Para este caso todo está asociado al ingreso per cápita del hogar.

Los valores que comprende este indicador se encuentran en el intervalo de 0 a 1 , siendo 0 el límite que indica la perfecta igualdad (los ingresos de todos los individuos son los mismos) y 1 la perfecta desigualdad (una persona se queda con todos los ingresos).

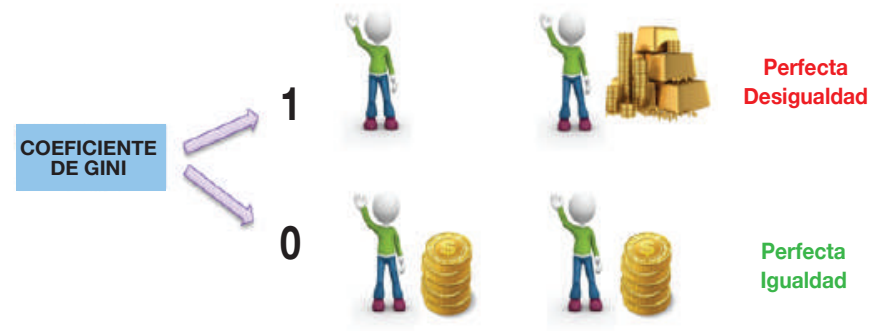

Fuente: Elaboración propia a partir de ENEMDU, pobreza y desigualdad (2015) Elaboración: Ing. Fernando Andrés Mayorga Núñez

El presente artículo detalla la evolución del coeficiente de Gini a nivel nacional clasificado por zonas urbana y rural, y en las principales ciudades del país; comparando los valores registrados en los meses de marzo y junio durante los periodos 2007-2015; 2008-2015 y 2014-2015.

Se han utilizado datos del Instituto Nacional de Estadística y Censos (INEC), los cuales se calculan a partir de la información registrada en la Encuesta Nacional de Empleo, Desempleo y Subempleo (ENEMDU), la misma que se levanta trimestralmente con una cobertura nacional, urbana y rural a partir de marzo de 2014, anteriormente la encuesta tenía sólo cobertura urbana en los meses de marzo y septiembre; y cobertura nacional, urbana y rural en los meses de junio y diciembre (INEC, Reporte de Pobreza por Ingresos, junio 2015, p.3).

\section{Coeficiente de Gini a nivel nacional}

En el mes de marzo la desigualdad económica a nivel del país refleja una disminución entre los años 2014 y 2015; el coeficiente de Gini pasa de 0,4791 a 0,4548; es decir una variación significativa de $-2,43$ puntos $(-5,07 \%)$.
Figura 1. Coeficiente de Gini a nivel nacional, mes de marzo, años 2014 y 2015

Coeficiente de Gini

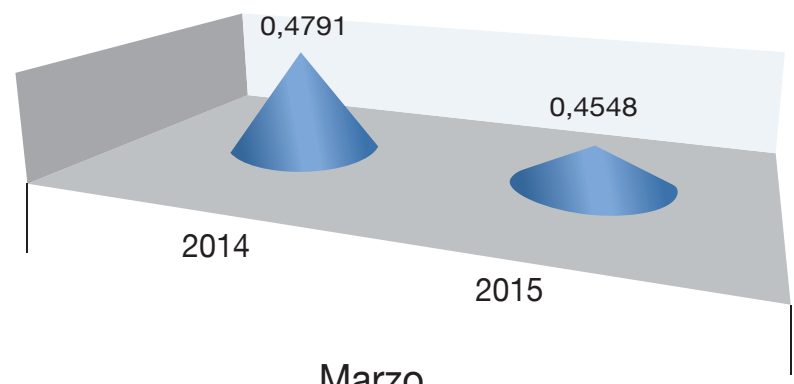

Marzo

Fuente: INEC - Encuesta Nacional de Empleo, Desempleo y Subempleo (ENEMDU. Pobreza y Desigualdad, Junio 2015
Elaboración: Equipo Técnico OBEST

Del 2007 al 2015, el coeficiente de Gini experimenta un decrecimiento en junio de cada año, a excepción del 2014 con un valor del 0,4858; así mismo registra un aumento en 2,26 puntos $(4,87 \%)$ con respecto al 2013 . Sin embargo, para el 2015 disminuye nuevamente, y el dato se sitúa en 0,4585; el más bajo del periodo analizado, es decir tuvo una variación de $-2,73$ puntos $(-5,61 \%)$ respecto al 2014 , siendo el valor más significativo.

Figura 2. Coeficiente de Gini a nivel Nacional, mes de Junio, periodo 2007-2015

- Coeficiente de Gini

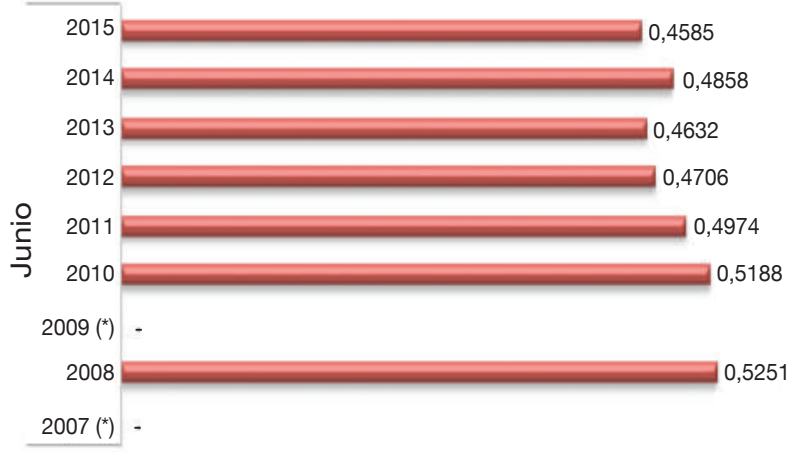

Fuente: INEC - Encuesta Nacional de Empleo, Desempleo y Subempleo (ENEMDU). Notas: $\left(^{(}\right)$La información en Junio 2007 y Junio 2009 solo fue representativa a nivel urbano Elaboración: Equipo Técnico OBES 


\section{Coeficiente de Gini a nivel nacional urbano.}

La zona urbana de un país se refiere a las áreas extensas que se encuentran dentro de la jurisdicción de un GAD, con una vasta población cuya actividad económica principal no es la agricultura, y además poseen características urbanas como por ejemplo: calles pavimentadas, alumbrado público o alcantarillado (UNICEF, 2012). De acuerdo a proyecciones realizadas por el INEC, la población urbana en el Ecuador para este año es de 10'326. 384 habitantes.

A nivel urbano, en marzo de cada año que corresponde al periodo 2008-2015, se observa que el coeficiente de Gini tiende a la baja desde el 2008 al 2010; a partir del 2011 se registran altas y bajas, siendo de mayor significancia las disminuciones del 2012 y 2015 con respecto a su año anterior, respectivamente. La variación en el 2012 fue de $-2,92$ puntos $(-6,11 \%)$, y en el 2015 de $-2,88$ puntos $(-6,25 \%)$.

Figura 3. Coeficiente de Gini a nivel nacional urbano, mes de marzo, periodo 2008-2015.

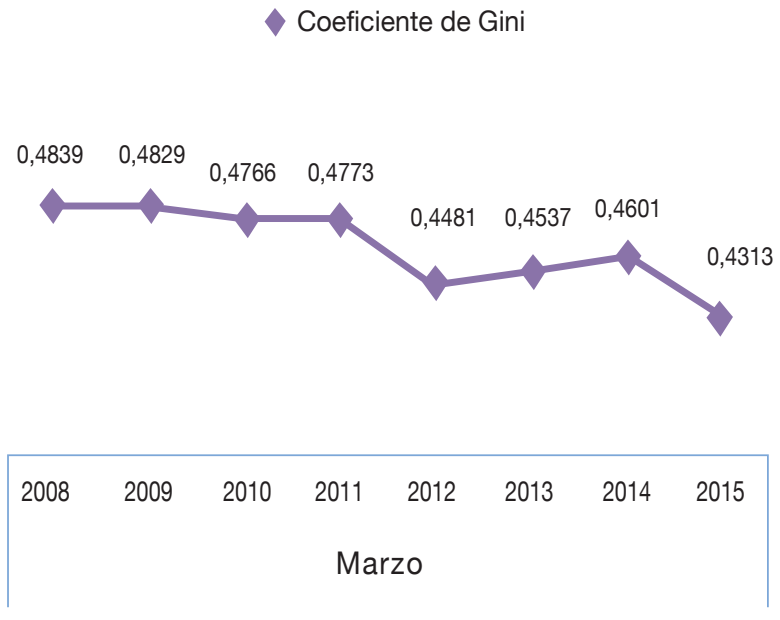

Fuente: INEC - Encuesta Nacional de Empleo, Desempleo y Subempleo (ENEMDU). Pobreza y Desigualdad, Junio 2015 Elaboración: Equipo Técnico OBEST

Para junio del periodo 2007-2015, aunque el coeficiente de Gini ha presentado incrementos en los años 2010 y 2014 respecto a su año predecesor, la tendencia dominante durante este periodo es decreciente. El valor más bajo registrado fue de 0,4421 en el 2013. Para junio del 2015 el indicador se ubicó en 0,4429; mostrando una variación de $-2,3$ puntos $(-4,93 \%)$ con relación al año 2014.
Figura 4. Coeficiente de Gini a nivel nacional urbano, mes de junio, periodo 2007-2015

- Coeficiente de Gini

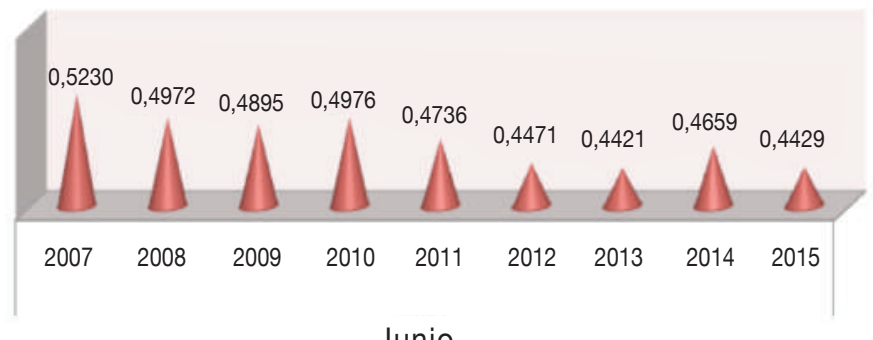

Junio

\section{Coeficiente de Gini a nivel nacional rural}

La zona rural de un país comprende áreas con características diferentes a las definidas en el área urbana. Según proyecciones hechas por el INEC, la población rural en el Ecuador para este año es de 5'952. 460 habitantes.

Entre marzo del 2014 y marzo del 2015, el coeficiente de Gini a nivel rural pasa de 0,4597 a 0,4521; es decir, registra una variación de $-0,76$ puntos $(-1,65 \%)$, la misma que no fue significativa.

Figura 5. Coeficiente de Gini a nivel nacional rural, mes de marzo, años 2014 y 2015

- Coeficiente de Gini

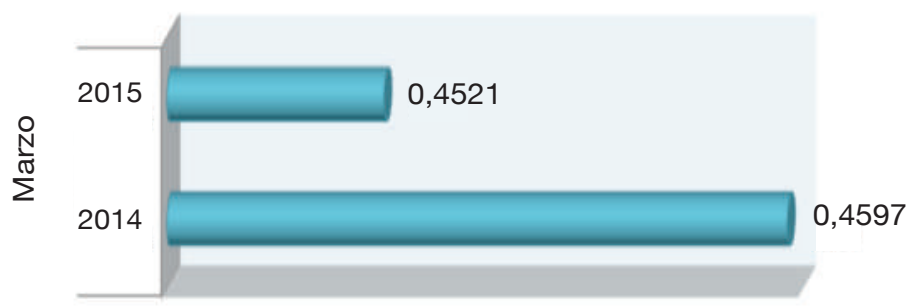


Con respecto al mes de junio, durante el periodo 2007-2015, el único año que presenta un incremento del coeficiente de Gini es el 2014, pues se ubica en 0,4591; registrando un incremento de 3,01 puntos $(7,01 \%)$ respecto al 2013 ; incremento que es significativo. Sin embargo, para el 2015, disminuye nuevamente a 0,4371 ; es decir, la variación respecto al 2014 se sitúa en $-2,2$ puntos $(-4,79 \%)$.

Figura 6. Coeficiente de Gini a nivel nacional rural, mes de junio, periodo 2007-2015

Coeficiente de Gini
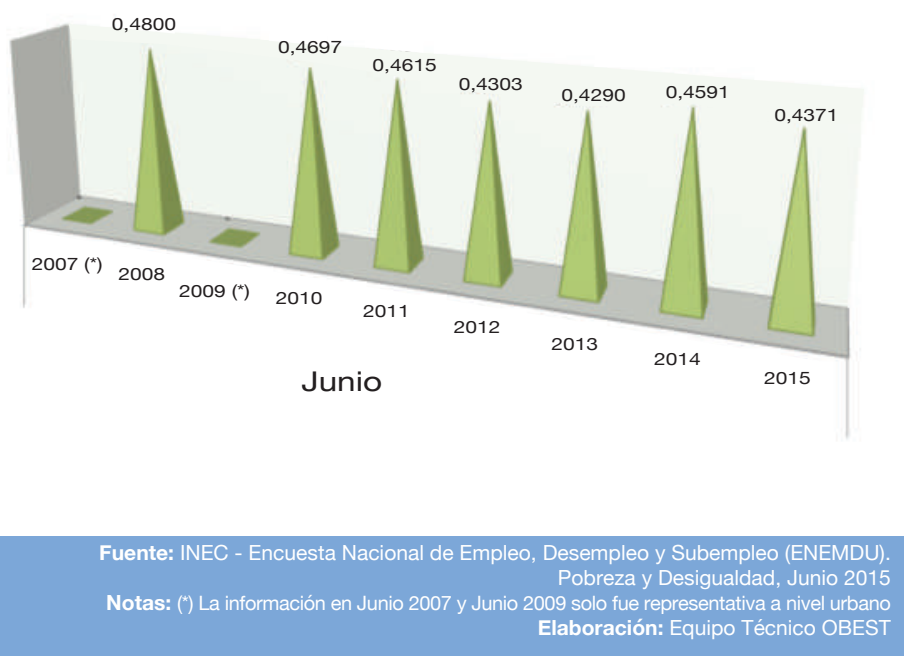

\section{Coeficiente de Gini en las principales ciudades del Ecuador}

A nivel de las principales ciudades del país según el INEC (Quito, Guayaquil, Cuenca, Machala y Ambato), entre marzo del 2014 y marzo del 2015 todas las ciudades a excepción de Cuenca presentan disminución en el coeficiente de Gini. En Cuenca se incrementa de 0,4246 a 0,4540; es decir su variación es de 2,94 puntos $(6,92 \%)$ respecto al 2014, la cual es significativa. Por el contrario, la ciudad que presenta mayor reducción en desigualdad económica, durante el mismo periodo, es Guayaquil; ya que su coeficiente de Gini pasa de 0,4118 a 0,3615 ; registrando una variación de $-5,03$ puntos $(-12,21 \%)$ con relación a marzo del 2014.

En el caso de Ambato, el coeficiente de Gini, durante el periodo 2008-2015, registra altos y bajos como las demás ciudades, pero la tendencia dominante es decreciente como se puede observar en la figura 7. En marzo del 2014 el coeficiente de Gini se ubicó en 0,4524 mientras que en marzo del 2015 disminuyó a 0,4242; la variación que tuvo fue de $-2,82$ puntos $(-6,23 \%)$. A pesar de esto, Ambato tuvo un coeficiente de Gini más alto que el de las otras ciudades, con excepción de Cuenca.
Figura 7. Coeficiente de Gini Ecuador, principales ciudades Periodo 2008-2015 (marzo)

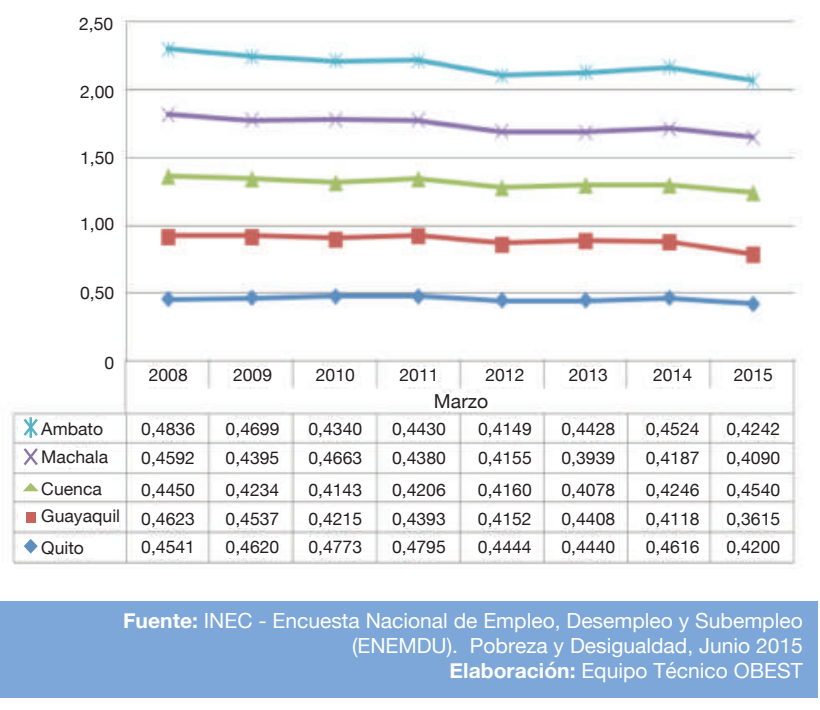

En el mes de junio durante el periodo 2007-2015, todas las principales ciudades del país presentan lecturas diferentes con disminuciones e incrementos en distintos años. En un comparativo entre 2014 y 2015, la ciudad que registra la reducción más notable del coeficiente de Gini es Machala, pasando de 0,4403 a 0,3893; es decir que la variación es de $-5,1$ puntos $(-11,58 \%)$. Cuenca por su parte, fue la única ciudad que registra un aumento en el coeficiente de Gini, de 0,4247 en junio del 2014 a 0,4254 en junio del 2015, pero éste no es significativo.

Ambato, en el mes de junio del año en curso, registra una disminución muy significativa en su coeficiente de Gini comparado con el 2014, de 0,4522 disminuye a 0,4198; siendo de -3.24 puntos $(-7,16 \%)$ la variación experimentada. Sin embargo, éste coeficiente es más alto que Machala y Guayaquil.

Figura 8. Coeficiente de Gini de las principales ciudades del Ecuador, mes de junio. Periodo 2007-2015.

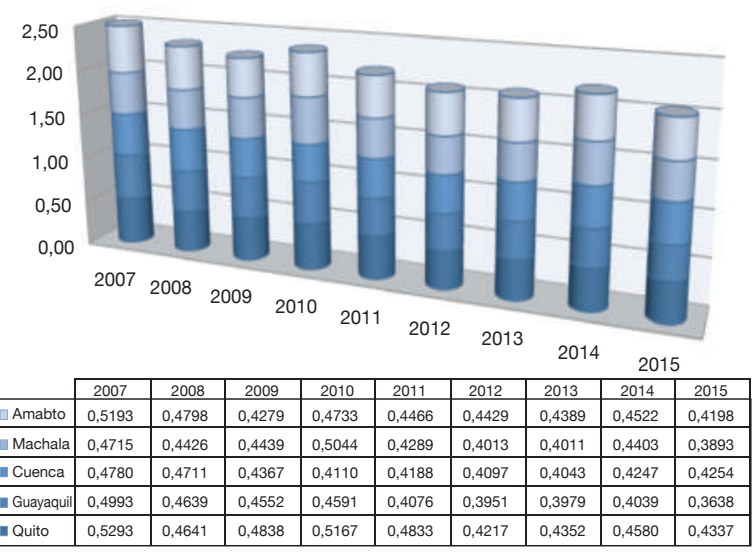

Fuente: INEC - Encuesta Nacional de Empleo, Desempleo y Subempleo (ENEMDU). Pobreza y Desigualdad, Junio 2015 Elaboración: Equipo Técnico OBEST 
En el 2015, Quito es la ciudad con mayor desigualdad económica de entre las cinco principales del Ecuador; mientras que Guayaquil es la que tiene menor desigualdad económica. Ambato se encuentra en la zona intermedia de este ranking, pero con más cercanía a las ciudades de coeficiente de Gini alto.

Figura 9. Coeficiente de Gini en las principales ciudades del Ecuador, Junio 2015

- Coeficiente de Gini

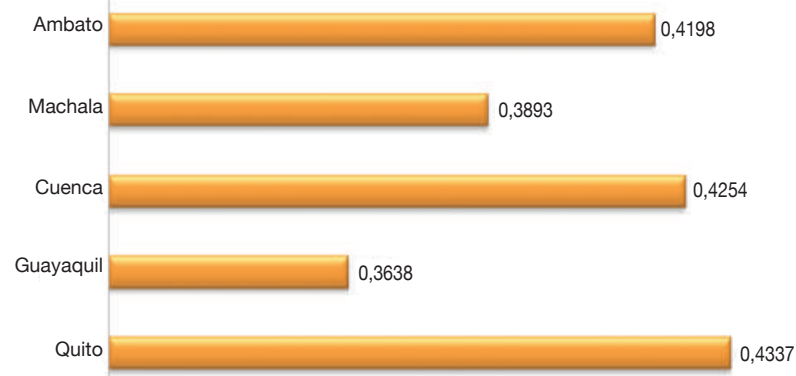

Fuente: INEC - Encuesta Nacional de Empleo, Desempleo y Subempleo (ENEMDU). Pobreza y Desigualdad, Junio 2015

\section{Referencias:}

- Banco Mundial (BM): http://datos.bancomundial.org/indicador/SI.POV.GINI

- Instituto Nacional de Estadística y Censos (INEC). (2015). Indicadores de Pobreza y Desigualdad Junio 2015. Recuperado de: http://www.ecuadorencifras.gob.ec/documentos/web-inec/PO-

BREZA/2015/Junio2015/Presentacion_pobreza_y_desiguald ad_junio15.pdf

- Instituto Nacional de Estadística y Censos (INEC). (2015).

Reporte de Pobreza por Ingresos Junio 2015. Recuperado de: http://www.ecuadorencifras.gob.ec/documentos/web-inec/PO-

BREZA/2015/Junio2015/Informe\%20pobreza\%20y\%20desi gualdad\%20junio15.pdf

- United Nations International Children's Emergency Fund (UNICEF). (2012). Niñas y niños en un mundo urbano.

Recuperado de: http://www.unicef.org/spanish/sowc2012/pdfs/SOWC\%202012\%20Executive\%20Summary\%20LoRes\%20PDF_SP_03132012.pdf

\section{Elaboración:}

Ing. Fernando Andrés Mayorga Núñez

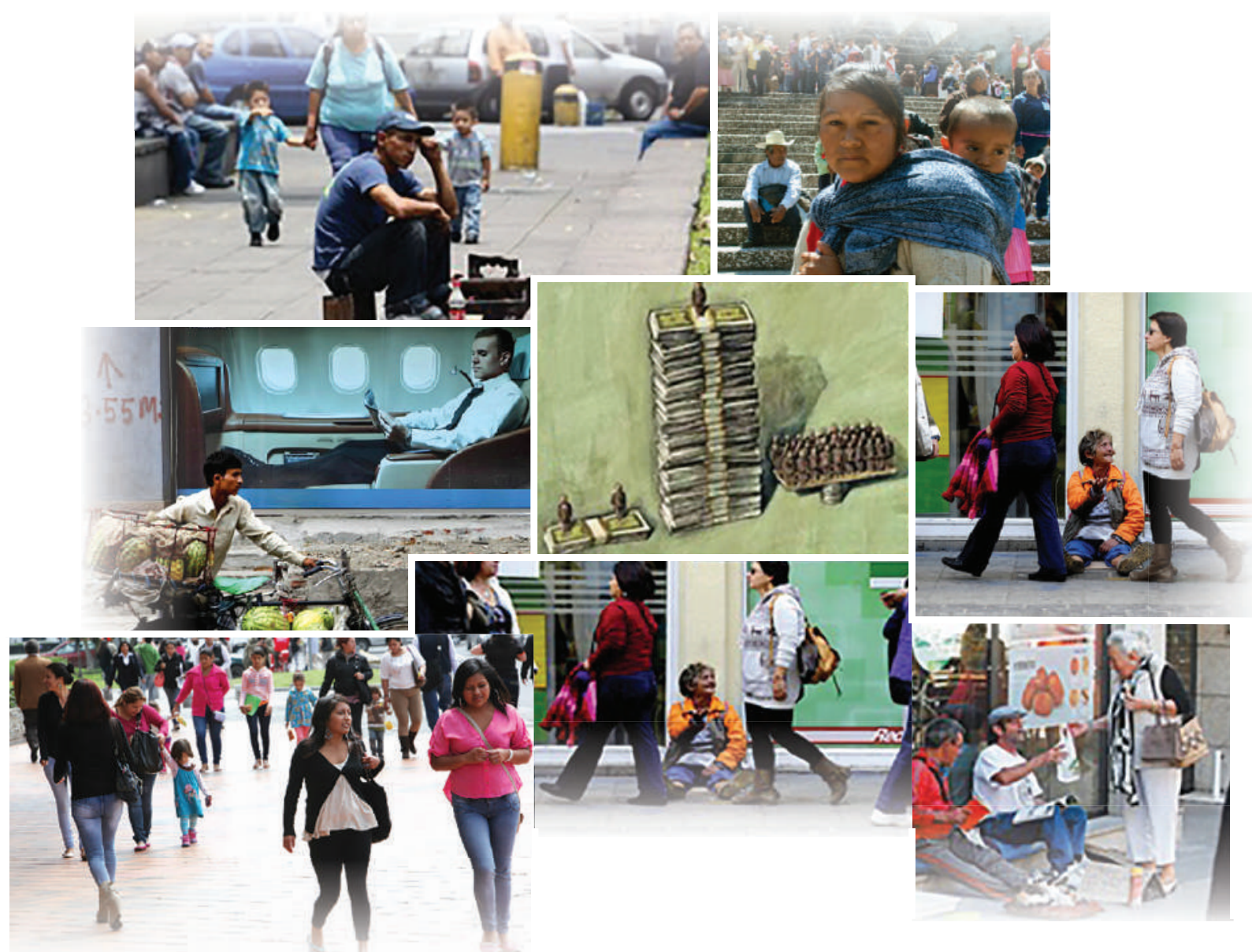

